\title{
NONEXISTENCE OF ORIENTATION REVERSING INVOLUTIONS ON SOME MANIFOLDS
}

\section{KYUNG WHAN KWUN ${ }^{1}$}

Motivated by a paper [1] of Olum, we point out a class of orientable closed manifolds of odd dimensions which do not admit any orientation reversing involution. Examples will follow the theorem and its proof. All manifolds are assumed to be connected and all orientable manifolds oriented.

Theorem. Let $M$ be a closed orientable manifold of dimension $2 n+1$ with the torsion subgroup of $H_{n}(M)$ cyclic of order $p, 2<p<\infty$. Then $M$ does not admit an orientation reversing involution.

Proof. Let $T$ be an involution of $M$. Then there exists an integer $k$ (unique modulo $p$ ) such that

$$
T_{*}: H_{n}(M) \rightarrow H_{n}(M)
$$

is given by $T_{*}(a)=k a$ for each torsion element $a \in H_{n}(M)$. Since $T_{*} T_{*}=$ identity, $k^{2} \equiv 1 \bmod p$.

By the functorial property of the universal coefficient sequence [2] of the cohomology, the restriction to the torsion subgroup of

$$
T^{*}: H^{n+1}(M) \rightarrow H^{n+1}(M)
$$

may be identified with

$$
\operatorname{Ext}\left(T_{*}, 1\right): \operatorname{Ext}\left(H_{n}(M), Z\right) \rightarrow \operatorname{Ext}\left(H_{n}(M), Z\right) .
$$

It then follows that for each torsion element $b$ of $H^{n+1}(M)\left(\cong H_{n}(M)\right)$, $T^{*}(b)=k b$.

Consider the commutative diagram

$$
\begin{gathered}
H^{n+1}(M) \stackrel{\cap \gamma}{\cong} H_{n}(M) \\
\cong \uparrow T^{*} \cong T_{* \gamma} \cong \downarrow T_{*} \\
H^{n+1}(M) \stackrel{\cap}{\longrightarrow}(M)
\end{gathered}
$$

where $\gamma \in H_{2 n+1}(M)$ is the orientation class. For each torsion element $b \in H^{n+1}(M)$,

Received by the editors February 10, 1969.

1 Supported in part by NSF Grant GP 8962. 


$$
\begin{aligned}
(\operatorname{deg} T)(b \cap \gamma) & =\left(b \cap T_{*}\right)=T_{*}\left(T^{*} b \cap \gamma\right)=T_{*}(k b \cap \gamma) \\
& =k^{2}(b \cap \gamma) .
\end{aligned}
$$

Hence $\operatorname{deg} T \equiv k^{2} \equiv 1 \bmod p$. Since $\operatorname{deg} T=1$ or -1 and $p>2, \operatorname{deg} T$ $=1$ and $T$ preserves orientation.

EXAMPLE 1 . Let $p, p^{\prime} \geqq 2$ be relatively prime integers and $M$ the connected sum $L(p, q) \# L\left(p^{\prime}, q^{\prime}\right)$ of two lens spaces. Or, more generally, let $M$ be the connected sum $L\left(p ; q_{1}, q_{2}, \cdots, q_{n}\right)$ $\# L\left(p^{\prime} ; q_{1}^{\prime}, q_{2}^{\prime}, \cdots, q_{n}^{\prime}\right)$ of generalized lens spaces (see [1] for definition) of dimension $2 n+1$ with $n$ odd. Then $M$ does not admit an orientation reversing involution.

Example 2. Let $M$ be the product $P_{2}(C) \times L(p, q)$ of the complex projective 2-space and a lens space with $p>2$.

Example 3. $L\left(p ; q_{1}, q_{2}, \cdots, q_{n}\right)$ does not admit an orientation reversing involution if $p \geqq 3$ and $n$ is odd. This fact also follows from a result [ 1 , Theorem V, p. 467] of Olum. On the other hand, if $p=2$ or $n$ is even, $L\left(p ; q_{1}, q_{2}, \cdots, q_{n}\right)$ does admit an orientation reversing involution.

\section{REFERENCES}

1. Paul Olum, Mappings of manifolds and the notion of degree, Ann. of Math. (2) 58 (1953), 458-480.

2. Edwin H. Spanier, Algebraic topology, McGraw-Hill, New York, 1966.

Michigan State University 